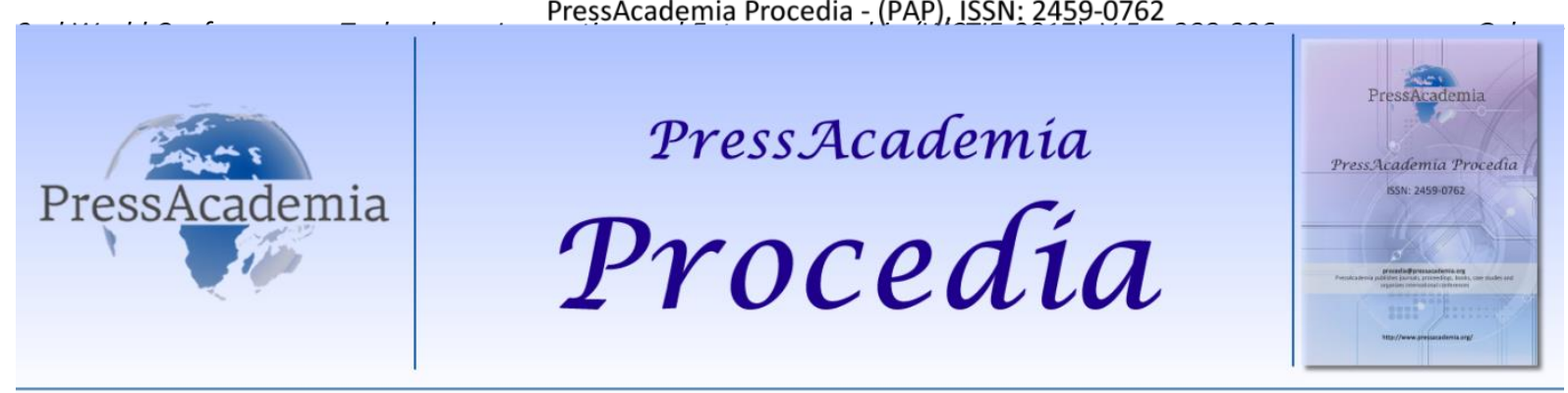

2nd World Conference on Technology, Innovation and Entrepreneurship

May 12-14, 2017, Istanbul, Turkey. Edited by Sefer Şener

\title{
APPLICABILITY OF RADIATION ABSORBING AND NON-ABSORBING NANOPARTICLES IN PHOTO- THERMAL ENERGY CONVERSION: A COMPARATIVE STUDY
}

\author{
DOI: 10.17261/Pressacademia.2017.615 \\ PAP-WCTIE-V.5-2017(53)-V.4-p.388-396
}

\section{Layth Al-Gebory}

Department of Mechanical Engineering and CEEE, Ozyegin University, Istanbul-Turkey. Department of Materials Engineering, University of Technology, Baghdad-Iraq. layth.ismael@ozyegin.edu.tr

\begin{abstract}
The radiative properties of nano-sized particles play a significant role in a widely range of industrial and engineering applications, such as chemical, electric power, industry, meteorology, biomedicine, biophysics, astronomy, combustion, fire and flame, as well as solar power plants. In the thermal applications field, nanoparticles are found to be an effective electromagnetic wave absorbers within UV-Visible wavelength range; where, short wavelength radiations include high energy photons. Because of their excellent and unique thermo-optical properties which are the basis of thermal phenomena and energy conversion, nanoparticles are used as working media in solar thermal collectors for photo-thermal energy conversion. The purpose of the present study is to investigate the applicability of absorbing (conducting) and non-absorbing (dielectric) nanoparticles in photo-thermal energy conversion. These particles are dispersed in a media to produce nanoparticle suspensions. The comparison between the radiative properties of the two nanoparticles with their suspensions is carried out. The radiative properties include scattering, absorption as well as extinction coefficients which are the most important parameters in the capture and utilization of solar energy. The contribution of particle scattering in the radiation attenuation is investigated by computing the single scattering albedo. Through the analysis of the radiative and thermal behaviour of the particulate medium, it is clear that photothermal conversion is important to not only the solar thermal systems, but also to the electricity generation and solar chemical technology, in addition to the other applications.
\end{abstract}

Keywords: Nanoparticles, radiative properties, photo-thermal conversion.

\section{INTRODUCTION}

The most common energy transport that we feel every day is the radiative energy. The energy travels from the Sun to the earth is in the form of electromagnetic radiations, this energy is scattered and absorbed as it goes through the atmosphere, then it is heating up everything around. Electromagnetic radiations allow us to see and feel everything in our surroundings as heat and light. In this operation, it is not only the strength and direction of radiative energy that are important, but also the interaction of the radiations with the objects and how it energizes our life.

Thermal radiation plays a signifcant role in many industrial and engineering applications, particularly at high temperatures. In the cases of convection and conduction, heat energy transfers between two objects depends on the temperature difference between these objects, the temperature difference may be slightly larger than one, and may reach two. In the thermal radiation transfer case which takes place between two distant objects depends on the difference between the fourth power of their absolute temperatures. If the materials properties are depend on the temperature and this included in the calculations, the radiative energy flux can be proportional to such a higher power of absolute temperatures, and as a result the importance of radiative energy transfer is dramatically enhanced at high temperatures. Because of this, radiation energy contributes significantly to energy transfer in combustion chambers, furnaces, rocket plumes, fires, high temperature heat exchangers, and during chemical explosions. therefore, the radiative energy transfer would improve the 
operation and design of such applications and devices. To this end, radiative energy transfer calculations need to be made strictly, which requires the utilize the accurate radiative properties.

Radiative energy transfer governs the distribution of temperature of the sun and emitted objects. Understanding this directional and spectral nature of radiative energy propagation is the basis for solar energy utilization. Given that we need more sustainable and cleaner energy production for the daily purposes and growing population of Earth, implementation of optimum and efficient radiation energy transfer principles for solar energy utilization and capture which have an important impact on the environment.

When an electromagnetic radiation or a photon interacts with a participating (particulate), the intensity of the incident radiation may be changed by scattering and/or absorption. One of the common examples of this interaction is absorption of sunlight by a cloud of smoke (which is a multitude of suspension of fine particles in air), another example is the scattering of sunshine by the atmosphere (molecules in the atmosphere, in fact, these are tiny particles) resulting in red sunsets and blue skies, and rainbow colors. Astrophysicists were the first dealt with radiation scattering by particles, and they were interested in the scattering of starlight by the dust. Scientists from other fields are concerned with the scattering of electromagnetic radiations: In the thermal field, photo-thermal energy conversion have been proposed by many researchers as one of the main energy sources. Therefore, solar power systems take a significant part of the research area, where the sustainable energy is needed.

Through the analysis of the solar energy utilization technology, it is clear that photothermal energy conversion which is important to not only the thermal utilization, but also solar chemical technology and the electricity generation. In solar thermal systems, solar thermal collector is the most important part in the photothermal conversion. In regular solar thermal collectors, surfaces of the evacuated tube and flat plate are heated by the incident solar radiation and then the thermal energy is transfered away by the working media (fluids) through convective heat transfer. The thermal resistant in converting the incident solar energy into thermal energy of fluids is large especially when vapor or gas is applied as the working media. Thus, the surface that absorbs the solar radiation has high temperature. One of the most important drawbacks of surface absorber is the limit thermal efficiency. Besides the surface absorber, volumetric solar absorption is another type of solar absorber in which the incident solar radiation is absorbed by a volume of fluid rather than the surface. Compared with surface absorbers, volumetric solar absorbers have many prominent advantages. Specifically, when a suspension of smal particles is used. Because the suspended particles are in contact with the base fluid, the incident solar energy absorbed by suspended particles can be efficiently transferred to the continues phase (surrounding fluid). Particles dispersed in liquid or gas have large surface areas to volume ratio that can enhance radiation absorption as well as convective heat transfer. Therefore, volumetric radiation energy absorbtion can efficiently operate under a high solar flux.

The progress in the nanotechnology and nanoscience during the past two decades or so that makes the application of nanoparticles in the solar thermal utilization. Specifically, the application of nanoparticle suspensions in solar thermal collectors which is proposed in different researchs. Indeed, nanoparticles are found to be an effective electromegnatic radiation absorbers at low wavelength range where short wavelength radiations included photons that carry high energy.

\section{LITERATURE REVIEW}

Radiative properties of nanoparticles and nanosuspensions have been widely investigated in the recent years, the effects of different nanoparticles types in addition to their physical and chemical characteristics on the radiative properties and radiative transfer phenomena have been observed.

The study of Lorenz predates that of Mie, this work proposed the general theory that describing radiative scattering by absorbing spherical particle, the theory known as the "Mie theory". Recently, in recognition of the contributions of Lorenz, the "Lorenz-Mie theory" becomes one of the most popular and reliable theory. The Lorenz-Mie theory cover the general scattering solution spherical particle (absorbing or non-absorbing) without limitation on particle size (Mie G. A. 1908).

An extensive reviews on thermal radiation phenomena in particulate suspensions have been introduced by Dombrovsky and Baillis (Dombrovsky et al. 2010), and also by Tien and Drolen (Tien et al. 1987).

Lord Rayleigh discussed radiation scattering and absorption by single spheres during the later part of the nineteenth century (Rayleigh 1964).

the impacts of dependent scattering were studied in a medium consisting of two spheres at an arbitrary distance between them and the index of refraction typical for that of soot particles (Ivezic et al. 1996). 
Dependent and independent scattering regimes were clarified for packed systems contain 10 spheres. A comparison study was carried out between the Foldy's effective field approximation (EFA) and the quasi crystalline approximation (QCA) for nanoparticle suspension in the Rayleigh scattering regime (Prasher 2005).

A hybrid methodology was introduced based on elliptically polarized light scattering (EPLS) to characterize metal oxide colloidal suspension (Aslan et al. 2006).

A comparison was done on cases include both fluid base absorbing and non-absorbing matrix, also for metal and non-metal particles (Wei et al. 2012).

A Theoretical method was developed for obtaining the radiative properties of nanoparticle suspensions with particle agglomeration. The microstructure of particle agglomeration groups have been generated using the diffusion limited cluster aggregation (DLCA) technique (Du et al. 2015).

The thermo-optical properties were investigated for nanoparticle suspensions for low temperature direct absorption solar thermal collectors (temperature between $00 \mathrm{C}$ and $1000 \mathrm{C}$ ) applications to study the potential of using suspended particles in solar power plants (Karami et al. 2016).

In the present study, the applicability of the absorbing (conducting) and non-absorbing (dielectric) nanoparticles in photothermal energy conversion is investigated. For that, two types of nanoparticles are proposed in the calculations, aluminum (Al) and alumina (Al2O3) nanoparticles, these nanoparticles are with particle size $d p=50 \mathrm{~nm}$ and suspended in a medium (water) to produce a nanosuspensions. The radiative properties (scattering, absorption as well as extinction coefficients) of the two nanoparticles types with their suspensions are calculated using the single scattering approximation technique based on the Lorenz-Mie theory. In addition, a comparison between the radiative properties of the two nanoparticles with their suspensions is carried out.

\section{DATA AND METHODOLOGY}

When incident radiations interact with participating (particulate) medium containing small particles, the intensity of the incident radiation may be changed by scattering and/or. How much it scattered or absorbed and into which direction an electromagnetic radiation passing through its vicinity depends on different parameters including (i) the shape and siz of the particle, (ii) the material of the particle (absorbing or nonabsorbing), (iii) the complex index of refraction, $\mathrm{m}=\mathrm{n}-\mathrm{ik}$, and (iv) the clearance (surface to surface distance) between particles (Modest 2012, Howell et al. 2016).

Different approaches have been considered to obtain the best fitted mathematical theory for describing the radiative properties of any particulate (participating) media. Light scattering by particles is characterized by the Rayleigh and the Lorenz-Mie theories. The Rayleigh scattering theory, after Lord Rayleigh, is applicable to dielectric (non-absorbing), spherical and small particle. On the other hand, the Lorenz-Mie theory, after Gustav Mie, is coverd the spherical particle (absorbing or non-absorbing) without limitation on particle size (Hahn 2009, Mishchenko 2014).

The conditions of the Rayleigh scattering theory are $\mathrm{x} \ll 1$ and $|\mathrm{m}| \mathrm{x} \ll 1$, where $x$ is the dimensionless size parameter $\left(x=\pi d_{p} / \lambda\right), m$ is the complex refractive index ( $\left.m=n_{\text {particle }} / n_{\text {media }}\right)$, and $n$ is the refractive index. Fig. (1) describes the radiative phenomenon in a particulate media. This figure shows an enclosure containing suspended particles in a medium. When the incident radiation travels through this media, it is attenuated by absorption and/or scattering, while other incident radiations are transmitted through this media to the other side. 


\section{Fig. (2) Incidence radiation on a particulate media}

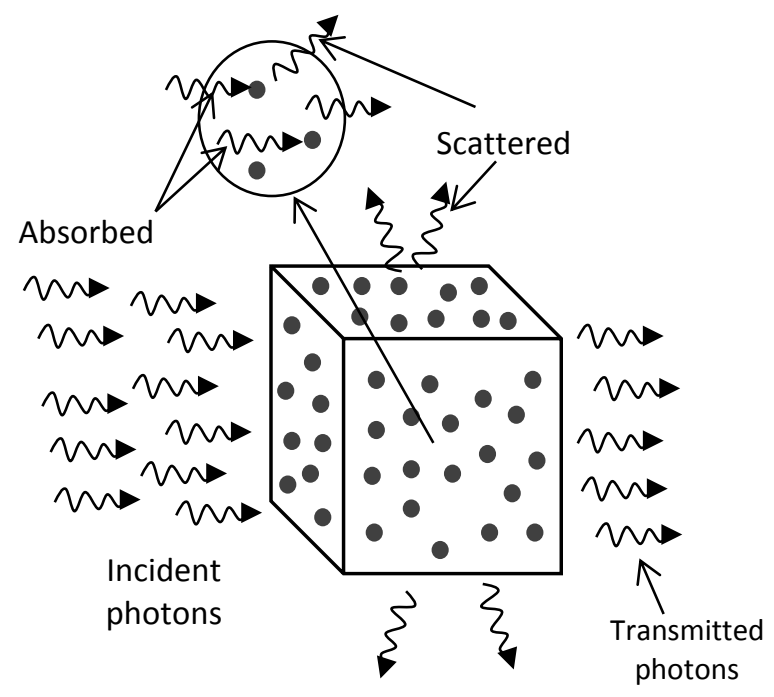

The Lorenz-Mie theory is proposed to present the interaction of incident radiation with a homogenous spherical particle. Based on such theory, the angular intensity functions $\left(i_{1}\right.$ and $\left.i_{2}\right)$ are describe the differential scattering cross sections, which are given by (Bohren et al. 1983, Mishchenko 2000):

$$
\begin{aligned}
& \sigma_{V V}=\frac{\lambda^{2}}{4 \pi^{2}} i_{1} \\
& \sigma_{H H}=\frac{\lambda^{2}}{4 \pi^{2}} i_{2}
\end{aligned}
$$

In these two equations, the subscripts $V V$ and $H H$ refer to vertically and horizontally polarized incident light. The radiative efficiencies $Q_{i}$ for the interaction of radiation with a particle of radius $r_{p}$ is defined as:

$$
Q_{i}=\frac{C_{i}}{\pi r^{2}}
$$

Where, $C_{i}$ is the cross sections normalized to the particle cross section $\pi r_{p}^{2}$, where $i$ refers for extinction $(i=e x t)$, scattering $(i=s c a)$ and absorption $(i=a b s)$. Then, considering the conservation of radiative energy, the extinction efficiency and cross section is represented as (Howell et al. 2016):

$$
\text { or } \begin{aligned}
Q_{\text {ext }, p} & =Q_{\text {scat }, p}+Q_{a b s, p} \\
C_{\text {ext }, p} & =C_{\text {scat }, p}+C_{a b s, p}
\end{aligned}
$$

In particulate suspensions with low particles volume fraction, the scattered intensity of a number of suspended particles is equal to the scattered intensity from a single particle multiple by the number of whole particles, which is known as the linear summation rule (SSA) (Howell et al. 2016). This rule is valid and suitable for most radiative transfer applications where independent scattering is considered. Then, the scattering and absorption coefficients of the suspended nanoparticles can be calculated as:

$$
\sigma_{\text {scat }}=N Q_{\text {scat }} \text { and } \sigma_{a b s}=N Q_{a b s}
$$

where, $N$ refers to the number of particles per unit volume which can be replaced by the particles volume fraction as following: 


$$
\varnothing=N V_{p}=N \pi \frac{d_{p}^{3}}{6}
$$

where, $V_{p}$ is the volume of the particle.

Then, the scattering and absorption coefficients become:

$$
\begin{aligned}
\sigma_{\text {scat }} & =\frac{3}{2} \frac{\emptyset\left(Q_{\text {scat }}\right)}{d_{p}} \\
\sigma_{a b s} & =\frac{3}{2} \frac{\emptyset\left(Q_{a b s}\right)}{d_{p}}
\end{aligned}
$$

The extinction coefficient (scattering+absorbtion) of the nanoparticles is as following:

$$
\sigma_{\text {ext }, p}=\sigma_{\text {scat }}+\sigma_{a b s}
$$

Yet, only the effect of the suspended particles on the radiative phenomena is demonstrated. While, any absorption effect comes from the medium should be included in the calculations. Therefore, the extinction coefficient of the medium is obtained from (Otanicar et al. 2009):

$$
\sigma_{\text {ext }, m}=\frac{4 \pi k_{\text {medium }}}{\lambda}
$$

where, $k_{\text {medium }}$ is the absorption index for the medium (water) that varies as a function of wavelength.

Then, the total nanoparticle suspension extinction coefficient when combined is as given below:

$$
\sigma_{\text {ext,total }}=\sigma_{\text {ext }, p}+\sigma_{\text {ext }, m}
$$

To explain the importance of the particle scattering behaviour in the total radiation attenuation, the single scattering albedo $\left(\omega_{\lambda}\right)$ is calculated, which is an important parameter specifically for non-absorbing (dielectric) particles:

$$
\omega_{\lambda}=\frac{\sigma_{\text {scat }, p}}{\left(\sigma_{\mathrm{abs}, \mathrm{p}+\mathrm{m}}+\sigma_{\mathrm{scat}, \mathrm{p}}\right)}=\frac{\sigma_{\text {scat }, \mathrm{p}}}{\sigma_{\text {ext,total }}}
$$

\section{FINDINGS AND DISCUSSIONS}

Two types of nanoparticles are under investigation in this study, Aluminium (absorbing particle) and alumina (nonabsorbing particle), the two nanoparticles at the same size $d_{p}=50 \mathrm{~nm}$ suspended in the base fluid (Water) with different particle volume fraction $(0.001,0.006$, and $0.01 \%)$. Therefore, the comparison in the radiative properties is carried out between two different particle types and with different particle concentration in the same medium. The radiative properties is the basis of the photothermal energy conversion, where the incident light intensity is converted to the thermal energy (the radiation is attenuated inside a media).

Fig. (2) shows the radiative efficiencies of the two nanoparticles types, the results obtained from the Lorenz-Mie theory. Zero absorption efficiency is obtained for the alumina $\left(\mathrm{Al}_{2} \mathrm{O}_{3}\right)$ nanoparticles, and aluminium (Al) particle shows a high scattering efficiency in comparison with that from the another particle. The radiative efficiency is inversely proportional with the wavelength, where nanoparticle is found to be an effective electromagnetic wave scattering and absorption at low wavelength (depending on te type of the particle). Aluminum particle shows high optical efficiencies in comparison with the second particle type $\left(\mathrm{Al}_{2} \mathrm{O}_{3}\right)$ particle at the same wavelength. 
(A)

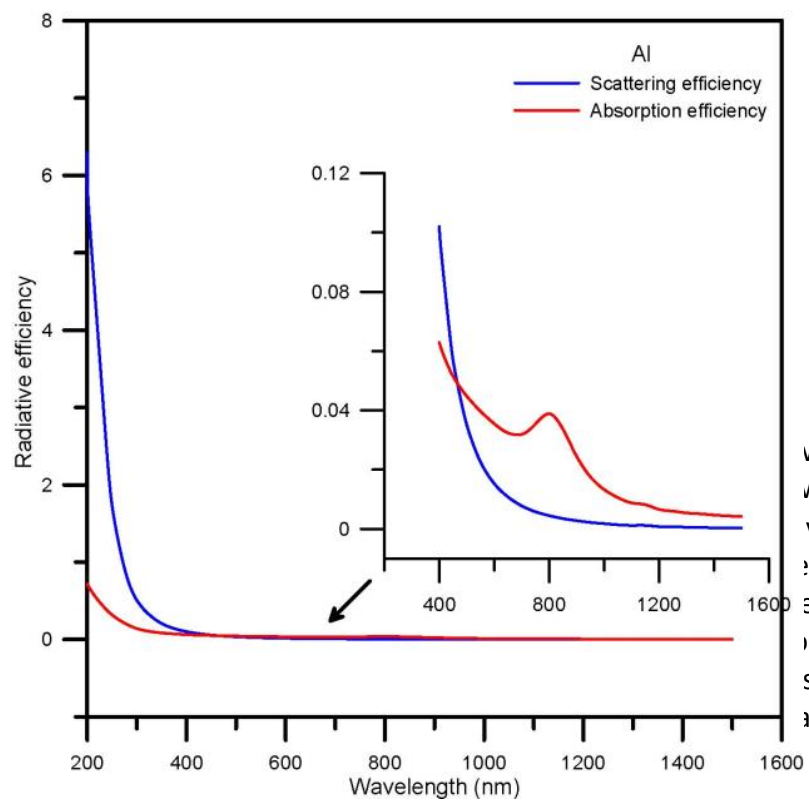

(B)

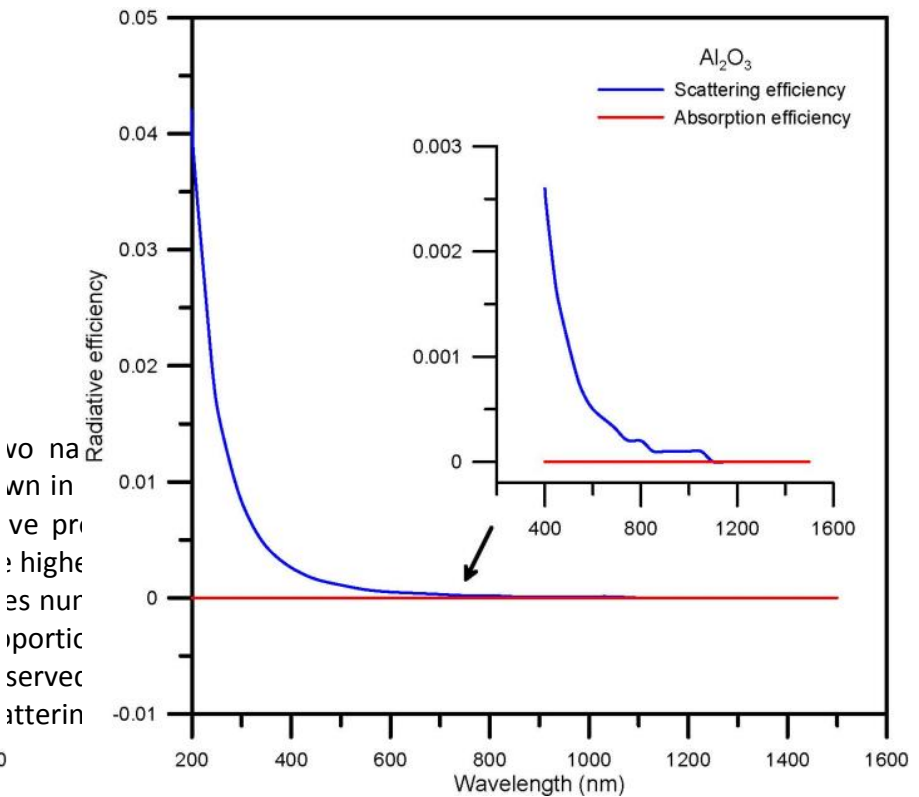

Fig. (2) Radiative efficiencies (scattering and absorption). (A) $\mathrm{Al}$ and (b) $\mathrm{Al}_{2} \mathrm{O}_{3}$ nanoparticles

Scattering phenomena tor particles in a participating media are attected by number of parameters including particle type, size, structure as well as the effect of the scattering from other particles in the same medium. The optical properties of a particle carries a significant impact on the radiative transfer phenomena.

(A)

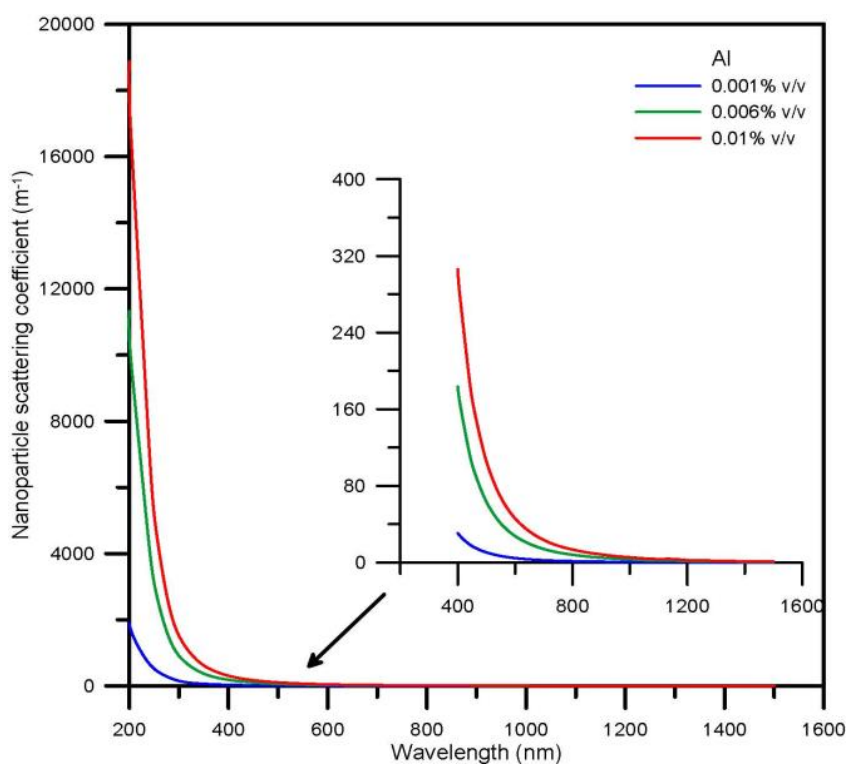

(B)

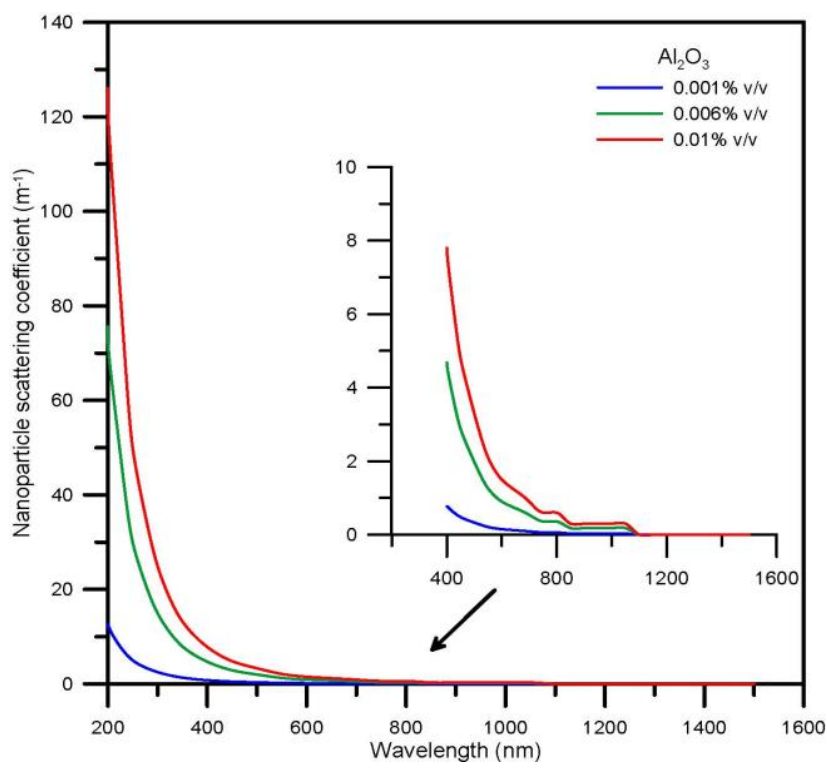

Fig. (3) Scattering coefficient for the nanoparticles. (A) $\mathrm{Al}$ and (b) $\mathrm{Al}_{2} \mathrm{O}_{3}$ nanoparticles 
The absorption coefficient of the Al nanoparticle is shown in Fig. (4). The same behaviour of the scattering coefficient, the absorption coefficient of the absorbing (Al) particle is inversely proportional to the wavelength. On the other hand, zero absorption coefficient is obtained for the non-absorbing $\left(\mathrm{Al}_{2} \mathrm{O}_{3}\right)$ nanoparticle. These are a result of the optical properties for the both nanoparticles, where each type of nanoparticles has its own optical (refractive and absorbtion) indices. The effect of particle concentration is also demonstrated in this figure.

Absorption coefficient play a signifcant role in the radiative transfer phenomena, which can be observed from the radiative transfer equation. In general, scattering and absorption coefficients of particulate media carries important impact in different applications. In thermal applications, these coefficients represent how mutch light is attenuated inside a medium.

Fig. (4) Absorption coefficient for (Al) nanoparticle

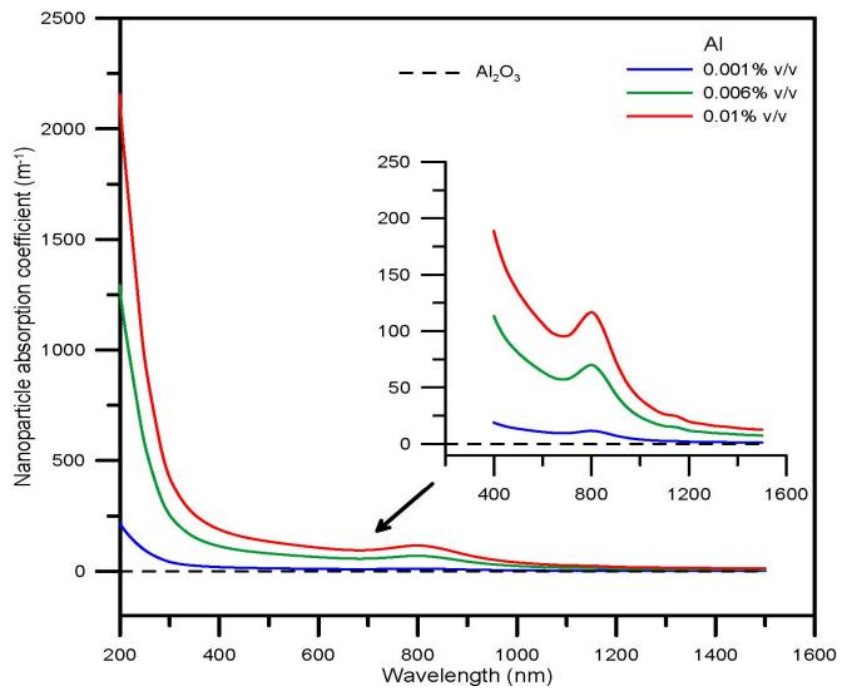

Fig. (5) shows the total extinction coefficient for the nanoparticle suspensions. The total extinction coefficient includes the effects of both nanoparticle and medium (water). The comparison between the two nanosuspensions types (water based $\mathrm{Al}$ and $\mathrm{Al}_{2} \mathrm{O}_{3}$ ) shows the importance of scattering and absorption coefficients of the nanoparticles in the radiation attenuation specifically at low wavelengths. While, the absorption coefficient of the medium play a significant role at longer wavelength (Infrared wavelength range). Radiation attenuation of a media indicates the photothermal energy conversion in such media.

Fig. (5) Extinction coefficient for the nanoparticle suspensions. Water based (A) $\mathrm{Al}$ and (b) $\mathrm{Al}_{2} \mathrm{O}_{3}$

(A)

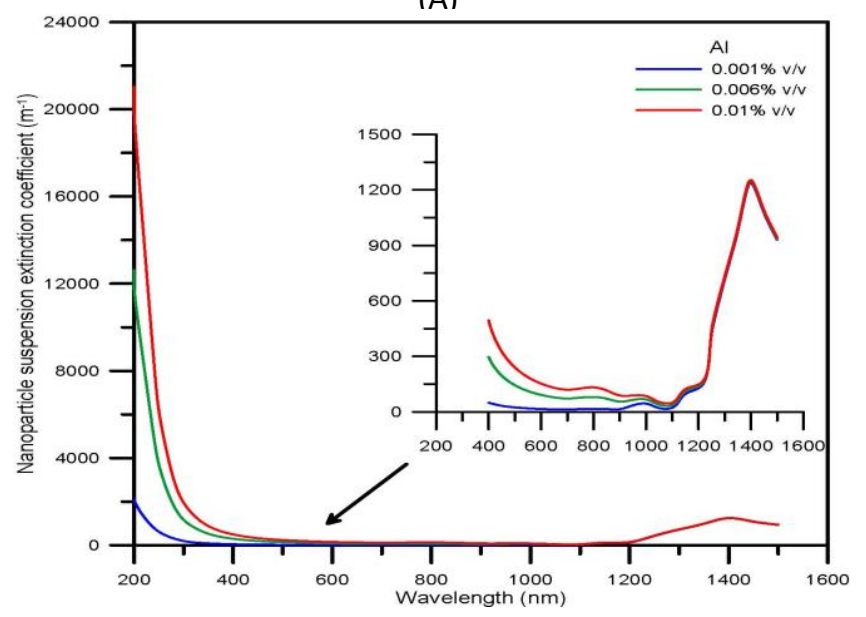
nanosusnensions

(B)

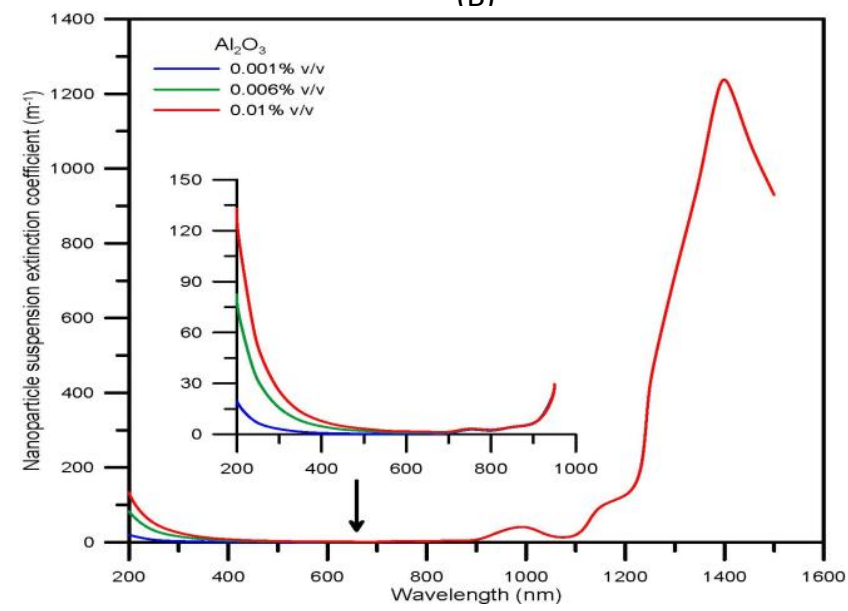


To explain the effect of the particle scattering behaviour on the total extinction coefficient, Fig. (6) shows the single scattering albedo for the nanoparticle types. A signifcant value of the single scattering albedo is observed for the water based $\mathrm{Al}_{2} \mathrm{O}_{3}$ nanosuspensions, where this particle is a non-absorbing partilce and only scattering from the particle is contributed in the total extinction coefficient. While, for the other nanoparticle (Al) low value of the single scattering albedo is obtained because the effect of the particle absorption is included in the total attenuation coefficient. In general, the single scattering albedo decreases as the wavelength becomes longer as a result of decreasing the scattering values.

Fig. (6) Single scattering albedo for the nanoparticle suspensions. Water based (A) $\mathrm{Al}$ and (b) $\mathrm{Al}_{2} \mathrm{O}_{3}$ nanosuspensions

(A)

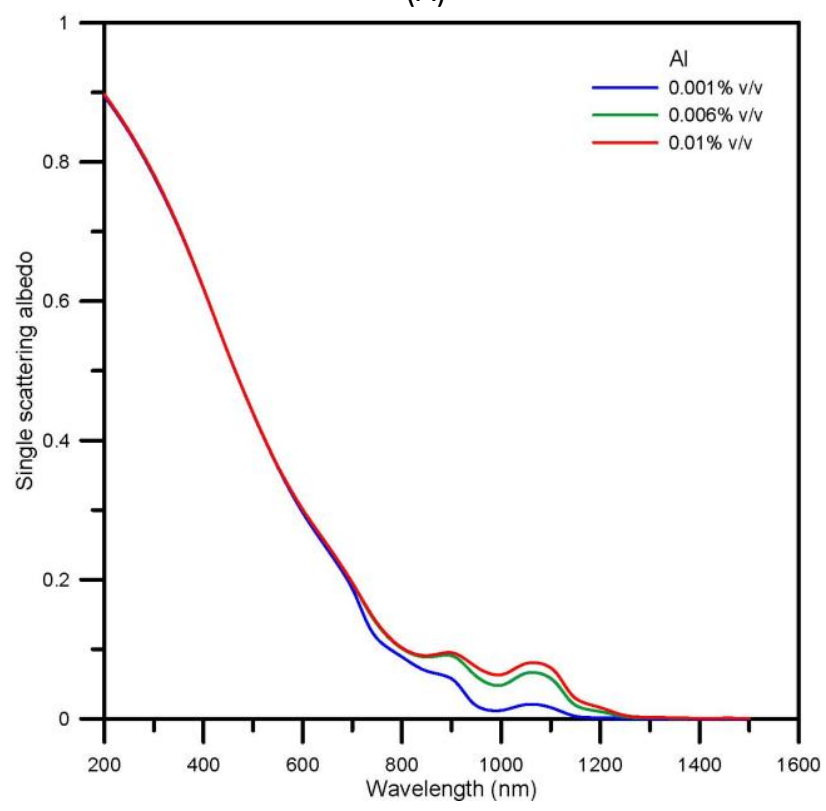

(B)

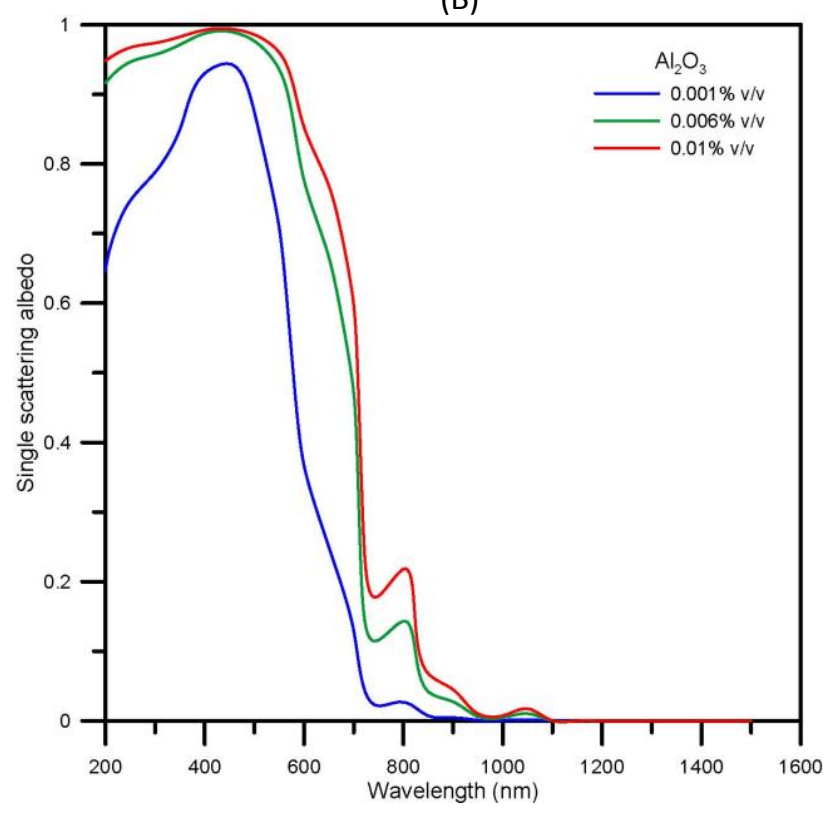

Nanoparticle are introduced for use in a wide range of industrial and engineering applications. Promising applications required suspended nanoparticles play a significant role in the thermal and optical properties at different wavelength ranges. Different nanoparticles have their own properties, and investigations are required to optimize the radiative properties for different nanoparticles (absorption and non-absorption nanoparticles). The present study, explored different radiative properties for these nanoparticles at different conditions (particle volume fraction) which is desired for a specific application including photothermal energy conversion.

Indeed, different radiative properties are obtained by changing particle concentration in the same medium, which is desired when a specified radiative properties is desired. In the field of photo-thermal energy conversion, optimizing the radiative properties of a participating medium is desired, where the amount of attenuated light represent the energy conversion in such media.

\section{REFERENCES}

Aslan M. M., Mengüç M. P., Manickavasagam S. and Saltiel C. (2006), "Size and shape prediction of colloidal metal oxide MgBaFeO particles from light scattering measurements", Journal of Nanoparticle Research.

Bohren C. F. and Huffman D. R. (1983), “Absorption and Scattering of Light by Small Particles”, Wiley-Inter science Publication, Canada.

Du M. and Tang G. H. (2015), “Optical property of nanofluids with particle agglomeration”, solar energy 122, 864-872.

Dombrovsky, L. A., and D. Baillis (2010), “ Thermal Radiation in Disperse Systems: An Engineering Approach”, Begell House, New York. 
Fan L. and Zhu C. (1998), "Principles of Gas-Solid Flows”, Cambridge University press.

Hahn D. W. (2009), "Light scattering theory”, Department of Mechanical and Aerospace.

Howell J R, Mengüç M P, Siegel R. (2016), “Thermal Radiation Heat Transfer”, 6th edition, CRC Press, New York.

Ivezic Z. and Mengüç M. P. (1996), "An investigation of dependent/independent scattering regimes using a discrete dipole Approximation", Int. J. Heat Mass TransJer. Vol. 39, No. 4, pp. 811-822.

Karami M., Akhavan -Behabadi M.A., Dehkordi M. R. and Delfani S. (2016), "Thermo-optical properties of copper oxide nanofluids for direct absorption of solar radiation", Solar Energy Materials and Solar Cells 144, 136-142.

Mie, G. A. (1908), “Beitr"age zur Optik tr “ uber Medien, speziell kolloidaler Metall"osungen”, Annalen der Physik, vol. 25, pp. 377-445.

Mishchenko M. I. (2014), “Electromagnetic Scattering by Particles and Particle Groups (An Introduction)”, $1^{\text {st }}$ Edition, Cambridge.

Modest M F. (2003), “Radiative Heat Transfer”, Academic Press-Elsevier science”, USA.

Otanicar T., Taylor R. A., Phelan P. E. and Prasher R. (2009), "Impact of size and scattering mode on the optimal solar absorbing nanofluid", Proceedings of the 3rd International Conference on Energy Sustainability, San Francisco, California, USA.

Prasher R. (2005), "Modification of Planck Blackbody Emissive Power and Intensity in Particulate Media Due to Multiple and Dependent Scattering", J. Heat Transfer 127(8), 903-910.

Rayleigh L. (1964), "On the light from the sky, its polarization and colour", Philos. Mag., vol. 41, pp. 107-120, 274-279, 1871, (reprinted in Scientific Papers by Lord Rayleigh, vol. I: 1869-1881, No. 8, Dover, New York.

Tien C. L., and Drolen B. L. (1987), "Thermal radiation in particulate media with dependent and independent scattering", in Annual Review of Numerical Fluid Mechanics and Heat Transfer, vol. 1, Hemisphere, New York, pp. 1-32.

Van de Hulst H. C. (1981), " Light Scattering by small particles", Dover publications, Inc., New York.

Wei W., Fedorov A. G., Luo Z. and Ni M. (2012), “Radiative properties of dense nanofluids”, Applied Optics, Vol. 51, No. 25.

Mishchenko M. I. (2000), "Light Scattering by Nonspherical Particles (Theory, Measurements and Applications)", academic press, USA. 